\title{
Recent Results from the High Altitude Water Cherenkov Obser- vatory
}

\author{
Kelly Malone for the HAWC Collaboration ${ }^{1, \star}$ \\ ${ }^{1}$ Physics Division, Los Alamos National Laboratory, Los Alamos, NM, USA
}

\begin{abstract}
The High Altitude Water Cherenkov (HAWC) Gamma-Ray Observatory, an extensive air shower detector consisting of 300 water Cherenkov tanks located at $4100 \mathrm{~m}$ in Puebla, Mexico, has been surveying the TeV gamma-ray sky for almost five years. HAWC can observe steady sources, variable sources, transients, which allows for probes of both astrophysical and particle physics phenomena. This includes the production and propagation of cosmic rays, studies of Lorentz invariance violation, and dark matter searches. I will discuss recent HAWC results as well as the future of the observatory.
\end{abstract}

\section{Introduction: Cosmic-ray origins and propagation}

It is a known fact that cosmic rays (CRs) are accelerated to $\sim 10^{20} \mathrm{eV}$ in nature [1], but which sources are responsible for this and the exact mechanisms are still largely open questions. CR acceleration is inherently interesting since the energies involved are much higher than can be reached by accelerators on Earth, but since cosmic rays are charged they bend in magnetic fields on their way to the Earth and it is somewhat difficult to pinpoint their origins.

There are advantages in studying gamma rays instead. CRs interact with their environment (such as the interstellar medium); neutral pions are created in this process. These neutral pions decay to gamma rays. Since gamma rays are not charged, they point back to sites of CR acceleration. This is the hadronic production mechanism of gamma rays.

Gamma rays are also produced via leptonic mechanisms. In the $\mathrm{TeV}$ range (discussed in this proceeding), the dominant mechanism is Inverse Compton scattering, in which a lower-energy photon scatters off an electron and has its energy boosted in the process.

Distinguishing between hadronic and leptonic gamma-ray sources (and thus identifiying which sources may be associated with cosmic rays) is a challenge in the field. For leptonic sources, the Klein Nishina component causes the gamma-ray emission to become suppressed starting at a few tens of $\mathrm{TeV}$, resulting in an energy-dependent spectral index and curvature in the spectra [2].

\section{Introduction to HAWC}

The High Altitude Water Cherenkov (HAWC) Observatory is an extensive air shower experiment with sensitivity to gamma rays with energies between a few hundred $\mathrm{GeV}$ and a few hundred $\mathrm{TeV}[3,4]$. It

\footnotetext{
^e-mail: kmalone@lanl.gov
} 

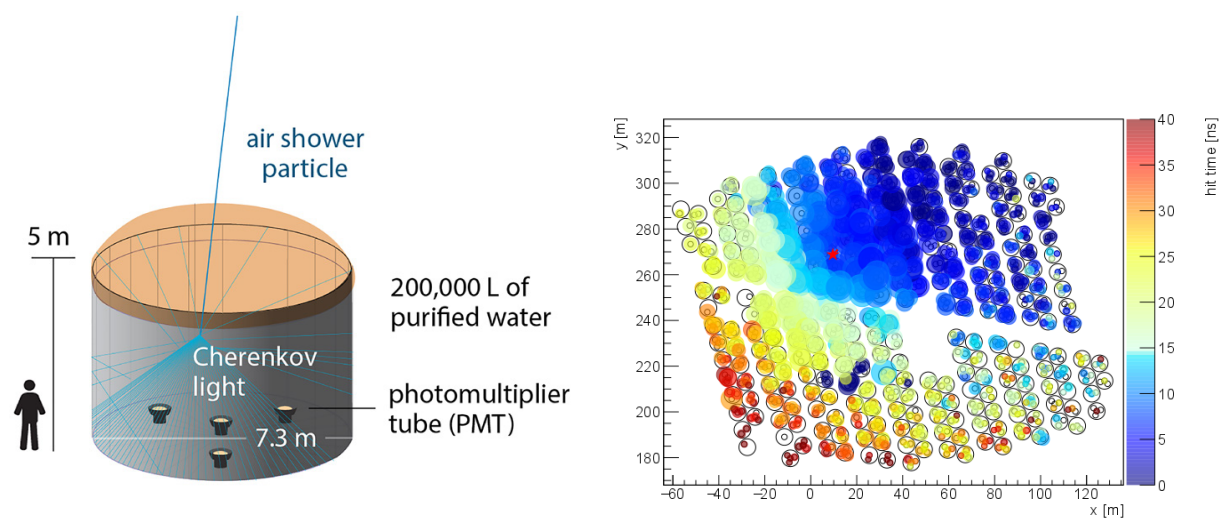

Figure 1: Left: A schematic of a tank. Right: The footprint of a detector as a high-energy event hits it. The colors denote time (with dark blue hitting earliest) and the size of the circles denotes the charge detected by each PMT. The shower core is denoted by a star.

is located at $19^{\circ}$ North on the saddle point between Pico de Orizaba and Sierra Negra in the state of Puebla, Mexico. The altitude is 4100 meters. HAWC has a duty cycle of $>95 \%$ and can observe $\sim 2 / 3$ of the overhead sky per day with an angular resolution of $\geq 0.1^{\circ}$. Partial science operations began in 2013, with full operations beginning in March 2015.

Extensive air shower (EAS) experiments work by detecting byproducts of the electromagnetic cascade induced when a gamma ray enters the Earth atmosphere and interacts with molecules in the air. This cascade consists of electrons, positrons, and lower-energy photons. Note that protons and other hadronic particles induce showers with a similar electromagnetic component, resulting in a very large backround for EAS arrays.

HAWC consists of an array of 300 tanks of water, each instrumented with four photomultiplier tubes (PMTs). These PMTs detect the Cherenkov light produced when the charged particles from the air shower pass through the tanks of water. Each tank is 5 meters high and 7.3 meters in diameter, and has 200,000 liters of water in a light-tight bladder. The entire footprint is 22,000 meters $^{2}$. Figure 1 shows a schematic of a HAWC tank.

As the EAS sweeps across the array, the timing of the PMT hits is used to reconstruct the direction and the charge is used to reconstruct the shower core and energy. The charge is also used for background rejection. Hadronic showers will have large depositions of charge far from the shower core due to the presence of muons, which have high transverse momenta. For more information on how HAWC operates, see [5].

\section{Recent Results}

HAWC has a far-reaching science agenda covering both Galactic and extragalactic observations as well as studies of fundamental physics phenomena and cosmic ray observations. Transients such as flaring sources and gamma-ray bursts are monitored. Additionally, there is an active follow-up program of multi-wavelength and multi-messenger phenomena.

One of the advantages of an EAS is the ability to perform an unbiased sky survey. The $2 \mathrm{HWC}$ (second HAWC) catalog was published in 2017 using 507 days of early HAWC data [6]. 39 sources 


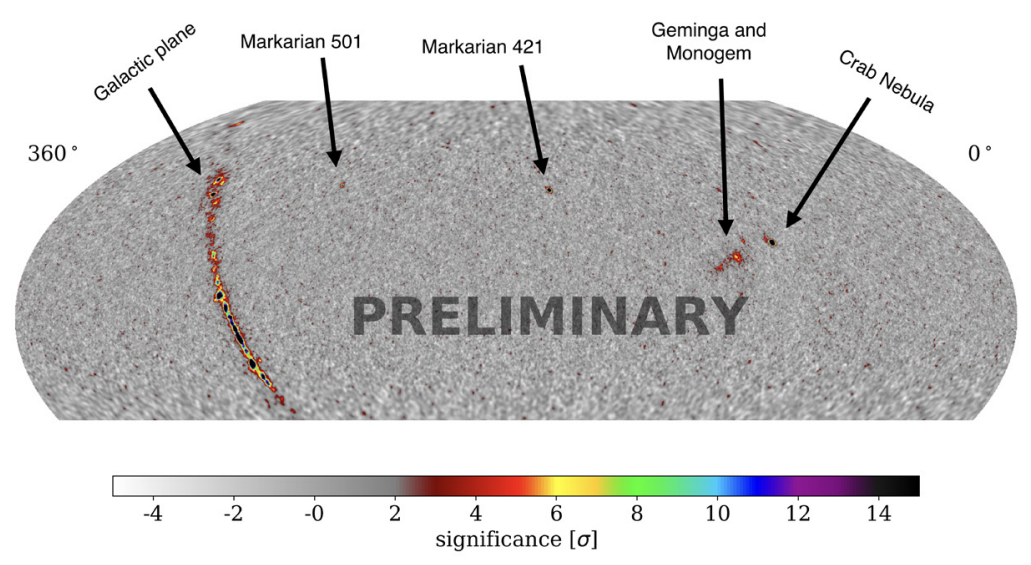

Figure 2: Significance map of the sky as seen by HAWC. 1346 days of data are used. Some prominent features are labeled.

were identified; 16 of them were new $\mathrm{TeV}$ discoveries. A handful have since been confirmed by other experiments [7]. Roughly half of the sources are unidentified (meaning that their nature cannot be determined using data from other wavelengths) while the rest are pulsar wind nebulae (PWNe), supernova remnants, and blazars.

Since the publication of the $2 \mathrm{HWC}$ catalog, additional data has been collected. Figure 2 shows an updated skymap with 1346 days of data ( 2.6 times that data published in the second catalog). Most of the emission is concentrated along the Galactic plane, although a few other sources are also visible. A few selected sources are discussed in the sections below.

\subsection{Galactic observations: Steady sources}

HAWC's wide field-of-view enables the observation of very extended sources ( $>1^{\circ}$ across). An example of this is HAWC's observations of the nearby (250-300 parsec) middle-aged ( $\sim 10^{5}$ year) PWNe Geminga and Monogem [8]. It has been previously hypothesized that contributions from nearby pulsars can explain the positron excess (originally observed by PAMELA [9]) beyond what is expected from CR interactions with the interstellar medium. In this scenario, gamma rays are produced when electrons and positrons diffuse and scatter on the cosmic microwave background.

The radial profiles of these sources show that Geminga and Monogem are indeed local sources of leptons, but that diffusion away from these sources is occurring on a slower timescale than previously was thought. Assuming that this slow diffusion constant persists over the entire distance between the Earth and the Geminga/Monogem region, pulsars are unlikely to be the origin of the positron excess. However, in the case of variable diffusion (as suggested by [10-12]), local pulsars are capable of producing the excess positrons. More studies of the region are needed.

HAWC has also studied less extended Galactic sources, such as the binary system SS 433 [13]. This source consists of a supergiant star accreting onto a compact object (either a black hole or a neutron star). This results in two perpendicular jets of plasma moving with a bulk velocity of $\sim 0.26 c$ [14]. The jets terminate inside the supernova remnant W50. Interactions inside this nebula accelerate charged particles, creating gamma rays. This is the first detection of spatially resolved 


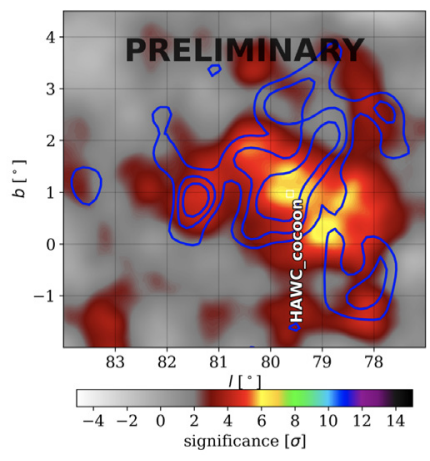

Figure 3: Significance map of the Cocoon region, after the nearby PWN has been removed. From [17]. A large-scale residual emission, roughly 2 degrees across, can be clearly seen.

$\mathrm{TeV}$-emitting lobes, and therefore the first direct proof of particle acceleration above $100 \mathrm{TeV}$ in jets. A leptonic origin of the $\mathrm{TeV}$ emission is favored over pure hadronic models [13]. Assuming protonproton collisions, the predicted gamma-ray flux is much less than what has been detected by HAWC. However, there are still open questions about how the acceleration occurs.

This is an important development because the jets studied here are thought to be scale models of more powerful jets in active Galactic nuclei (AGN), which are likely the sources of the highest-energy CRs. Since AGN are extragalactic objects, we are not able to spatially resolve them at this time.

Recent improvements to HAWC's data analysis chain have allowed the extension of the dynamic range of the experiment to above $100 \mathrm{TeV}$, resulting in the highest energy source catalog to date [15]. There are nine Galactic sources that emit at very high significance $(>5 \sigma)$ above $56 \mathrm{TeV}$, eight of which are within a degree of the Galactic plane (the ninth source is the Crab Nebula). Curiously, all of the eight inner Galactic plane sources are coincident with high spin-down pulsars $\left(\dot{E}>10^{36} \mathrm{erg} / \mathrm{s}\right)$. Each of these sources also remains extended in apparent size above $56 \mathrm{TeV}$. This is unexpected, as their spatial coincidence with pulsars implies leptonic emission mechanisms. The high-energy electrons that create $>56 \mathrm{TeV}$ gamma rays are expected to cool fairly quickly, so assuming an injection of electrons from the center of each source, it is puzzling to see how they have diffused such distances before cooling. Three of the nine $>56 \mathrm{TeV}$ sources have significant emission continuing above 100 $\mathrm{TeV}$. These are the highest-energy collection of sources ever detected in any astrophysical messenger.

One of the most interesting regions that emits above $56 \mathrm{TeV}$ is the Cygnus Cocoon region. The emission seen by HAWC is coincident with a lower-energy region detected by the Fermi-LAT telescope [16]. The Fermi observation was attributed to a cocoon of freshly accelerated cosmic rays. HAWC observations extend this cocoon to higher energies. The Cocoon region is fairly complicated in the TeV regime, as there is also the PWN of PSR J2032+4127 and the $\gamma$-Cygni SNR. Once these two sources are subtracted, a large-scale $\left(\sim 2^{\circ}\right)$ residual emission (the Cocoon) is visible [17]. Various possible hadronic models for this emission are currently being explored. See Figure 3 for an image of the region.

\subsection{Extragalactic observations: Bursts and transients}

HAWC also monitors the extragalactic sky. To date, two sources have been detected: Markarian 421 and Markarian 501 [6]. 
HAWC is continuously scanning the sky for new extragalactic sources (both steady ones and transients). The flare monitor program provides real-time daily monitoring of known blazars for flares [18]. In the event of a detection, observatories capable of performing in-depth followups are automatically notified, which can give multi-wavelength pictures of flaring sources. It is designed to detect flares with timescales of minutes to days.

HAWC also monitors the sky for short-term bursts. This includes the gamma ray burst (GRB) program. The sky is monitored in real-time for bursts with durations of less than 100 seconds. HAWC is also capable of performing archival data analysis of GRBs detected by other observatories.

HAWC also has several active programs that emphasize multi-messenger observations. HAWC receives alerts from IceCUBE about PeV neutrino events and from LIGO about gravitational wave events. Both time-dependent (around the time of the alert) and time-integrated (using archival data) analyses are possible. In the event that an alert covers a region that is, at the time of the event, out of HAWC field-of-view, an analysis is completed as soon as the source moves into HAWC's field of view. HAWC has placed limits on both electromagnetic counterparts to gravitational waves [19] and astrophysical neutrinos.

\subsection{Fundamental physics}

In addition to observing Galactic and extragalactic astrophysical objects, HAWC probes fundamental physics phenomena such as Lorentz invariance violation (LIV) and dark matter.

Detections of extremely-high-energy photons ( $>100 \mathrm{TeV}$, like the ones discussed in Section 3.1), lead naturally to studies of LIV. LIV can modify the particle dispersion relation. In some superluminal LIV models, this modification is predicted to lead to a decay of photons above some critical energy over short distances. Over astrophysical distances, this decay probability is predicted to be $100 \%$, which would lead to hard cutoffs in astrophysical spectra. HAWC observations above $100 \mathrm{TeV}$ limit the LIV energy scale to $>10^{31} \mathrm{eV}$, or 800 times the Planck energy scale [20, 21].

This analysis is performed by finding the energy above which the spectrum of an astrophysical source is inconsistent with both background and events who have mis-reconstructed energies by inserting a hard cutoff (step function) into the spectrum. The likelihood value for the nominal spectral fit is compared to the likelihood of the version with the hard cutoff inserted to determine the highest energy that that gamma rays are significantly detected at. The most significant high-energy source is 2HWC J1825-134. At the 95\% confidence level, photons of 250 TeV are detected [21].

HAWC's wide field-of-view also means that there are many targets to search for indirect signatures of dark matter annihilation and decay. Optimal sensitivity is achieved for dark matter masses between 1 and $100 \mathrm{TeV}$. Examples of targets include the Andromeda galaxy, dwarf spheroidal galaxies, the Sun, and the Galactic halo [22].

\section{HAWC upgrade and future}

HAWC is on its way to achieving many of its stated science goals. An "outrigger array" has recently been installed [23]. This consists of smaller WCD tanks (each with only one PMT) placed around the main array, with coarser spacing. This breaks the degeneracy between large showers with cores far from the array and smaller showers falling closer to the array. With an increase in effective area of approximately a factor of four, this will lead to a predicted increase in sensitivity of at least a factor of two above $10 \mathrm{TeV}$.

Planning has also begun for a new wide-field of view TeV experiment in the Southern Hemisphere [24]. This would allow for a better view of the Galactic center as well as provide full-sky 
coverage for transients. The Southern Wide-field-of-view Gamma Observatory (SWGO) Collaboration (www.swgo.org) has recently been formed, and sites in Chile and Argentina, among others, are under active consideration.

\section{References}

[1] M. Tanabashi et al. (Particle Data Group), Phys. Rev. D 98, 030001 (2018)

[2] R. Moderski, M. Sikora, P.S. Coppi, F. Aharonian, Monthly Notices of the Royal Astronomical Society 363, 954 (2005)

[3] A.U. Abeysekara, A. Albert, R. Alfaro, C. Alvarez, J.D. Álvarez et al., The Astrophysical Journal 843, 39 (2017)

[4] A.U. Abeysekara, A. Albert, R. Alfaro, C. Alvarez, J.D. Álvarez et al., The Astrophysical Jorunal 881, 134 (2019)

[5] A.J. Smith, Proceedings of Science (34th International Cosmic Ray Conference) 966 (2015)

[6] A.U. Abeysekara, A. Albert, R. Alfaro, C. Alvarez, J.D. Álvarez et al., The Astrophysical Journal 843, 40 (2019)

[7] A.U. Abeysekara, A. Archer, W. Benbow, R. Bird, R. Brose et al., The Astrophysical Journal 866, 24 (2018)

[8] A.U. Abeysekara, A. Albert, R. Alfaro, C. Alvarez, J.D. Álvarez et al., Science 358, 911 (2017)

[9] O. Adriani, G.C. Barbarino, G.A. Bazilevskaya, R. Bellotti, M. Boezio et al., Nature 458, 607 (2009)

[10] D. Hooper, I. Cholis, T. Linden, K. Fang, Physical Review D 96, 103013 (2017)

[11] S. Profumo, J. Reynoso-Cordova, N. Kaaz, M. Silverman, Physical Review D 97, 123008 (2018)

[12] K. Fang, X.J. Bi, P.F. Yin, Q. Yuan, The Astrophysical Journal 863, 30 (2018)

[13] A.U. Abeysekara, A. Albert, R. Alfaro, C. Alvarez, J.D. Álvarez et al., Nature 562, 82 (2018)

[14] S. Fabrika, Astrophysics and Space Physics Reviews 12, 1 (2004), astro-ph/0603390

[15] A.U. Abeysekara, A. Albert, R. Alfaro, J.R. Angeles Camacho, J.C. Arteaga-Velázquez et al., Phys. Rev. Lett. 124, 021102 (2020)

[16] M. Ackermann, M. Ajello, A. Allafort, L. Baldini, J. Ballet et al., Science 334, 1103 (2011)

[17] B. Hona, H. Fleischhack, P. Huentemeyer, Proceedings of Science (36th International Cosmic Ray Conference) 699 (2019)

[18] A.U. Abeysekara, R. Alfaro, C. Alvarez, J.D. Álvarez, R. Arceo et al., The Astrophysical Journal 843, 116 (2017), 1704.07411

[19] I. Martinez-Castellanos, Proceedings of Science (36th International Cosmic Ray Conference) 737 (2019)

[20] H. Martínez-Huerta, A. Pérez-Lorenzana, Physical Review D 95, 063001 (2017)

[21] H. Martinez-Huerta, S. Marinelli, J.T. Linnemann, J. Lundeen, Proceedings of Science (36th International Cosmic Ray Conference 738 (2019)

[22] J. Lundeen, J.P. Harding, Proceedings of Science (36th International Cosmic Ray Conference) $528(2019)$

[23] V. Joshi, A. Jardin-Blicq, Proceedings of Science (35th International Cosmic Ray Conference) 806 (2017)

[24] P. Abreu, A. Albert, R. Alfaro, C. Alvarez, R. Arceo et al., The Southern Wide-Field GammaRay Observatory (SWGO): A Next-Generation Ground-Based Survey Instrument, in Bulletin of the AAS (2019), Vol. 51, p. 109, 1907.07737 